\title{
PARITY NONCONSERVATION IN THE HYDROGEN ATOM
}

\author{
R.R. LEWIS and W.L. WILLIAMS \\ Harrison M. Randall Laboratory of Physics, \\ University of Michigan, Ann Arbor, Michigan 48104, USA
}

Received 3 September 1975

\begin{abstract}
We investigate the possibility of observing parity nonconscrvation with a metastable hydrogen atom beam, by analyzing two effects which should permit detection of the $2 \mathrm{~S}-2 \mathrm{P}$ admix ture predicted by the Weinberg model.
\end{abstract}

The discovery of unified theories of electromagnetism and weak interactions [1] has opened many new questions about the properties of leptons [2]. One can reasonably ask what observable effects are predicted for the hydrogen atom [3]. This atom has "parity multiplets" with nearly degenerate levels of opposite parity and is particularly sensitive to parity nonconserv. ing (PNC) interactions between the electron and nucleus. Further it enjoys the great advantage that its properties are completely calculable. We have analyzed two effects which offer hope of measuring the PNC ( $T$-invariant) interaction coupling constants in hydrogen and deuterium.

The predicted effect of the weak interactions is to couple the $2 \mathrm{~S}_{1 / 2}$ and $2 \mathrm{P}_{1 / 2}$ states of the hydrogen atom yielding an admixture of these states of order

$|\delta|=\left|\left\langle H_{\text {weak }}\right\rangle\right| / \Delta E \approx \frac{1 / 80 \mathrm{~Hz}}{\delta} \approx 10^{-11}$,

where $\delta \approx 1058 \mathrm{MHz}$ is the $2 \mathrm{~S}_{1 / 2}-2 \mathrm{P}_{1 / 2}$ separation (Lamb shift). Such an admixture imposes the need for very great sensitivity in any new parity test. The small size of the weak PNC matrix element demands that it be observed in interference with some parity conserving (PC) matrix element. Practical matters have led us to consider the possible tests that could be made on an intense metastable hydrogen beam in vacuum.

Since atoms interact rather strongly with their environment, we should consider the possible enhancement of PNC effects by suitable external perturbations. To begin with, the levels in $n=2$ can be shifted by a magnetic field and made to cross, with the real parts of their energy differences vanishing. At about $575 \mathrm{G}$ the levels $\beta\left(2 \mathrm{~S}_{1 / 2}, m_{j}=-\frac{1}{2}\right)$ and $\mathrm{e}\left(2 \mathrm{P}_{1 / 2}, m_{j}=+\frac{1}{2}\right)$ cross and at about $1190 \mathrm{G}$ the levels $\beta$ and $\mathrm{f}\left(2 \mathrm{P}_{1 / 2}, m_{j}=-\frac{1}{2}\right)$ cross. This perturbation does not itself mix states of opposite parity but it can enhance the effect of weak interactions by reducing the energy denominator $(\Delta E-\mathrm{i} \Gamma / 2)^{-1} .(\Gamma \approx 100 \mathrm{MHz}$ is the width of the $2 \mathrm{P}$ states.) The admixture near a crossing is increased by a factor of 21 .

If the hyperfine states with $m_{I}= \pm \frac{1}{2}$ are labeled by \pm , respectively, we find at $1190 \mathrm{G}$ an admixture between two of the four nearby states

$$
\begin{aligned}
& \left\langle\mathrm{f}_{-}\left|H_{\text {weak }}\right| \beta_{-}\right\rangle=-0.013 \mathrm{i}\left(C_{1}+C_{2}\right) \mathrm{Hz}, \\
& \left\langle\mathrm{f}_{+}\left|H_{\text {weak }}\right| \beta_{+}\right\rangle=-0.013 \mathrm{i}\left(C_{1}-1.11 C_{2}\right) \mathrm{Hz},
\end{aligned}
$$

where $C_{1}$ and $C_{2}$ are the coupling coefficients of the spin independent and spin dependent PNC interactions, respectively. Any PNC effect sensitive to the hyperfine components can therefore be used to measure $C_{1}$, which is the same for each component, and $C_{2}$, which changes sign.

At $575 \mathrm{G}$ a more subtle result is found ${ }^{\ddagger}$. There is only one non-vanishing weak matrix element coupling the nearby levels, and it arises solely from the spin dependent terms

$\left\langle\mathrm{e}_{-}\left|H_{\text {weak }}\right| \beta_{+}\right\rangle=-0.025$ i $C_{2} \mathrm{~Hz}$.

The cancellation of $C_{1}$ is an "accident" for the pure Coulomb potential, resulting from the hyperfine separations, as well as the magnetic moments, having the same ratio, 3 , in $2 \mathrm{~S}_{1 / 2}$ and $2 \mathrm{P}_{1 / 2}$. As a consequence, any PNC effect resonant at $575 \mathrm{G}$ determines $C_{2}$ alone. This is of particular significance since this parameter is otherwise difficult to measure.

\footnotetext{
\# The possibility of PNC effects at this crossing was first mentioned by Robiscoe [4].
} 
Next we consider the effect of electric perturbations. The matrix element of the electric dipole interaction $V=e E \cdot r$, where $E$ is the applied electric field, can admix many of the same states as the weak interaction, and thus offers an opportunity for producing interference with the weak PNC admixture. Both electric and weak perturbations can induce parity admixtures, but they can be distinguished from one another by the difference in their transformation properties, at least insofar as the electric field can be reversed accurately during the experiment. The electric field transforms like a vector under rotations, and $H_{\text {weak }}$ like a scalar.

This difference in multipole order also raises a fundamental difficulty in producing the desired interference. The reduced matrix elements of $H_{\text {weak }}$ are pure imaginary, while those of $V$ are real. Both operators are even under time reversal, but the different multipole order brings down an extra factor $i$, resulting in a phase difference and in an apparent absence of interference.

We have found two different ways of producing the desired interference effect. One is to observe the Lyman $-\alpha$ decay radiation produced by a sudden entry into an electric field parallel to a $575 \mathrm{G}$ magnetic field, which results in a coherent superposition of the two states $\beta$, e. Here the desired extra phase is built up by the propagation of the two states with slightly different energies. In this case both parity admixtures have the same energy denominator, whose phase and modulus do not influence the interference. Another way is to measure the circular dichroism in the absorption line $2 S \rightarrow 3 S$, in an electric field perpendicular to an 1190 $\mathrm{G}$ magnetic field. This gives a non-resonant Stark admixture of $\beta$ and $e$, and a resonant weak admixture of $\beta$ and $\mathrm{f}$. In this case the weak admixture acquires an extra factor $i$ as well as the enhancement from the resonant energy denominator.

The first effect requires passing the beam through a small hole in a pair of charged parallel plates in the magnetic field. The sudden approximation is valid for a $10 \mathrm{keV}$ beam passing through a $1 \mathrm{~mm}$ hole. Metastable atoms in the state $\beta_{+}$enter the magnetic field adiabatically, remaining in the same state. On suddenly passing through the electric field, each atom is put into a superposition of the long-lived and short-lived states $\beta_{+}, \mathrm{e}_{-}$. The amplitude of the $2 \mathrm{P}$ state $\mathrm{e}_{-}$in the beam downstream from the plates is, to within an overall phase,

$$
\left[\epsilon \rho \exp (\mathrm{i} \varphi) \exp \left\{+\mathrm{i}\left(1-\frac{4}{3} b\right) t\right\} \exp \left(-\frac{1}{2} \gamma t\right)+\mathrm{i} \operatorname{Im} \delta\right]
$$$$
\times\left(1-\frac{4}{3} b+i \gamma / 2\right)^{-1} \text {. }
$$

Our notation is such that all frequency parameters are measured in units of the Lamb shift $\delta \approx 1058 \mathrm{MHz}$; thus $b=\mu_{0} B / \delta=(B / 756 \mathrm{G}), \delta=\left(H_{\text {weak }}\right) / \delta, \epsilon=$ $\left.\sqrt{3} e E a_{0} / \delta\right)=(E / 477$ volt $/ \mathrm{cm})$, etc. The "regeneration amplitude" $\epsilon \rho \mathrm{e}^{\mathrm{i} \varphi}$ is a parameter characteristic of the strength and extent of the electric field. Both $\rho$ and $\varphi$ depend in a known way on the magnetic field, $\rho$ being even about the line center and $\varphi$ being odd. Because of the added complex factors, the square of the above amplitude will contain an interference term between $\epsilon$ and $\delta$. Any experiment measuring the $2 \mathrm{P}$ state probability, such as the decay rate to the ground state, will show this interference. Consequently, a detector sensitive to Lyman- $\alpha$ coming from the region downstream of the regenerator should show a small contribution proportional to $\operatorname{Im} \delta / \epsilon \rho$, which can be made of order $10^{-8}$. Observation of this small contribution can be accomplished by a triple subtraction technique since the interference term changes sign with $E$, with $B$ and is odd about the line center. This effect can be used to measure $C_{2}$ in hydrogen and deuterium at the $575 \mathrm{G}$ crossing.

The second effect can be observed by measuring the absorption of circularly polarized light by atoms in the beam as they pass through crossed electric and magnetic fields. A CW dye laser tuned to the energy difference between the states $\beta\left(2 S_{1 / 2}, m_{j}=-\frac{1}{2}\right)$ and $\beta\left(3 S_{1 / 2}, m_{j}=-\frac{1}{2}\right)$ is required. This particular component of the Balmer- $\alpha$ line is well resolved from the other components, especially the strong $2 \mathrm{~S}-3 \mathrm{P}$ components. The width of the absorption line $2 \mathrm{~S}-3 \mathrm{~S}$ is determined mainly by the width of the $3 \mathrm{~S}$ state, $\Gamma(3 S) \approx 1 \mathrm{MHz}$, and so the hyperfine structure is well resolved.

In the absence of perturbations, the $2 \mathrm{~S}-3 \mathrm{~S}$ absorption line can only result from magnetic dipole transitions, which are too weak to observe. The introduction of an electric field produces an admixture of $n S$ and $n \mathrm{P}$ states, giving an electric dipole amplitude $\propto E$ and an absorption $\propto E^{2}$. If parity is conserved there should be no difference in this absorption for right and left circularly polarized light, at least not for light incident perpendicular to the beam axis.

If the effect of weak PNC interactions is included, it gives rise to different absorption rates $R_{ \pm}$for photons with helicity \pm 1 . This yields a circular dichroism (CD) 
$\mathrm{CD}=\frac{R_{+}-R_{-}}{R_{+}+R_{-}}=\frac{2}{9} \frac{\gamma b \operatorname{Im} \delta}{\epsilon}\left[\left(1-\frac{2}{3} b\right)^{2}+(\gamma / 2)^{2}\right]^{-1}$,

which has the value at resonance $(b=3 / 2)$

$\mathrm{CD}_{\text {resonance }}=\frac{\dot{4} \operatorname{Im} \delta}{3 \varepsilon \gamma} \approx 14 \operatorname{Im} \delta / \epsilon$.

This circular dichroism is a very sensitive test of parity since it can be observed by a triple subtraction of the rate of absorption, first with reversal of the circular polarization, then with a reversal of the sign of the electric and of the magnetic fields.

We are confident that in spite of the small size of the predicted PNC effects in the hydrogen atom, it is feasible to measure them in beams experiments. This offers the hope of measurement of $C_{1}$ and $C_{2}$ both for hydrogen and for deuterium, and thus of a complete determination of the weak neutral coupling constants of electrons with nucleons. Further work is in progress and a detailed discussion will be forthcoming. Experiments to investigate both of the effects discussed here have been initiated in collaboration with T.B. Clegg from the University of North Carolina.

We have profited considerably from conversations on this subject with Min-Shih Chen, Gerald Feinberg and P.G.H. Sandars.

\section{References}

[1] S. Weinberg, Rev. Mod. Phys. 46 (1974) 255.

[2] G. Feinberg and M.Y. Chen, Phys. Rev. D10 (1974) 190; J. Bernabeu, T.E.O. Ericson and C. Jarlskog, Phys. Letters 50B (1974) 467;

M.A. Bouchiat and C.C. Bouchiat, Phys. Letters 48B (1974) 111;

I.B. Khriplovich, JETP Letters 20 (1974) 315.

[3] Ya.B. Zel'dovich, JETP 36 (1959) 964;

F. Curtis Michel, Phys. Rev. 138B (1965) 408.

[4] R.T. Robiscoe, Phys. Rev. 138A (1965) 22. 